\title{
Crash Risk Prediction Model of Lane-Change Behavior on Approaching Intersections
}

\author{
Yingshuai Li, ${ }^{1,2,3}$ Jian $\mathrm{Lu},{ }^{1,2,3}$ and Kuisheng $\mathrm{Xu}^{3}$ \\ ${ }^{1}$ Jiangsu Key Laboratory of Urban ITS, Southeast University, Si Pai Lou \#2, Nanjing 210096, China \\ ${ }^{2}$ Jiangsu Province Collaborative Innovation Center of Modern Urban Traffic Technologies, Southeast University, Si Pai Lou \#2, \\ Nanjing 210096, China \\ ${ }^{3}$ School of Transportation, Southeast University, Si Pai Lou \#2, Nanjing 210096, China
}

Correspondence should be addressed to Jian Lu; lujian_1972@seu.edu.cn

Received 8 March 2017; Accepted 6 July 2017; Published 9 August 2017

Academic Editor: Cengiz Çinar

Copyright (C) 2017 Yingshuai Li et al. This is an open access article distributed under the Creative Commons Attribution License, which permits unrestricted use, distribution, and reproduction in any medium, provided the original work is properly cited.

The driving tendency of drivers is one of the most important factors in lane-changing maneuvers. However, the heterogeneity of the characteristics of drivers' lane-changing behaviors has not been adequately considered. The primary objective of the present study is to explore the risk level of the lane-changing implementation process under different driving tendencies upon approaching signalized intersections in an urban area. This paper defines the Integrated Conflict Risk Index (ICRI), which takes into account the probability and severity of risk. Using the index as the dependent variable, the risk prediction model of implementing the lanechange process is established. A series of experiments, which included a questionnaire, a number of tests, and on-road experiments, was conducted to identify the driving tendencies of the participants. A combination of video recording and instrumented vehicles was used to collect lane-changing trajectory data of different driving tendencies. The parameters of the model were calibrated, and the results indicate that driving tendency has a significant effect on the risk level of lane-changing execution. More specifically, the more aggressive the driving tendency, the higher the risk level. The quantitative results of the study can provide the basis for conflict risk assessment in the existing lane-changing models.

\section{Introduction}

The design and assessment of traffic safety management are difficult in real transportation because of the cost and risks of collecting trajectory data. Therefore, microscopic traffic simulation models are powerful tools extensively used to evaluate traffic safety management policies and to assess their effects. The effectiveness of the results depends on the accuracy of the models. In microscopic traffic simulation models, car following and lane changing are two fundamental components. Compared to car following models, in which vehicles are in the same lane and the driver behavior is only influenced by the lead vehicle, the lane-changing process involves a high level of interaction between the vehicles and is more complex [1-3]. As a result of the importance of the role of microscopic traffic simulation models, how to improve the accuracy of the lane-changing model has attracted wide attention among scholars over the past decades. A large amount of work has been conducted to collect trajectory data [4-9] and formulate models of lane changing [10].

Despite the large number of lane-changing models that has been published, many previous studies have focused on freeways [11] in order to analyze the impact on traffic capacity and traffic safety. Other researchers have been interested in lane-changing behaviors at freeway on-ramp merging areas, where the maneuver is mandatory. Limited research has been reported regarding lane-changing behaviors along arterial streets, where drivers may change lanes more frequently. Only a few researchers have studied lane-changing maneuvers on urban streets [12], and even these models, according to their research reports, do not involve the impacts of driver characteristics because of the scarcity of reliable data [13]. 


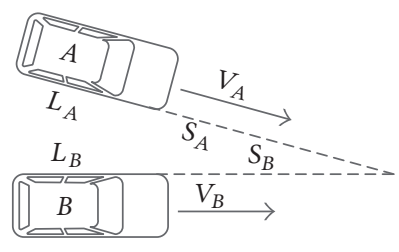

(a)

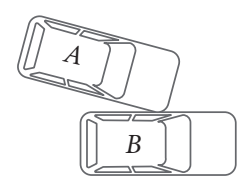

(b)

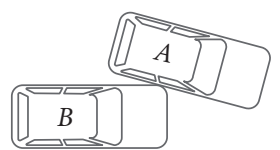

(c)

FIGURE 1: The lane-change conflict process diagram.

Driver characteristics and reliable vehicle trajectory data are the two most important factors in lane-changing models $[14,15]$. Driver characteristics include driving tendency and driving skill level. Vehicle trajectory data include the position, speed, and acceleration of the subject vehicle and the vehicles ahead of and behind the subject vehicle in the current lane, as well as in the adjacent lanes. Currently, there are two methods for data collection for the lane-changing process [9]: video recording and instrumented vehicles. Video recording can record any lane-changing model parameters and requires detailed trajectory data, which can help to analyze the relationship between the subject vehicle and surrounding vehicles. However, this method is limited in terms of location and length of road. In addition, it is difficult to extract driver characteristic information. The instrumented vehicle method can record driver characteristic information, and, compared to video recording, can improve the precision of vehicle trajectory data. Unfortunately, it is too difficult to extract the trajectory data of surrounding vehicles.

Apart from the lack of research on lane-changing maneuvers on urban streets, the models for these maneuvers do not consider driver characteristics and the scarcity of reliable data. Another downside of existing models is that they ignore the process of executing lane-changing maneuvers and only deal with the decision-making process [13]. They simply assume a straight line from the starting point of the current lane to the ending point of the target lane for a fixed lanechanging duration. This assumption is far from realistic lane-changing behavior. In real life situations, traffic flow characteristics are highly affected by the execution process of a lane-changing maneuver. To improve the microscopic traffic simulation results, the execution process of a lanechanging maneuver must be considered.

The primary objective of the present study is to explore the risk level of the lane-changing implementation process under different driving tendencies upon approaching signalized intersections in an urban area. In order to achieve this objective, we need four steps: (1) to establish an integrated index for evaluation of conflict risk; (2) to identify the driving tendency of the participants; (3) to extract the driving trajectory data of participants with different driving tendencies; (4) to propose a traffic conflict risk prediction model to predict the security risk of lane-changing. The results of the study can provide the basis for conflict risk in the existing lanechanging models and thus improve the accuracy of these models.
The methodology for establishing a risk prediction model is presented in the next section. The questionnaire survey, test, and experiment design for data collection are presented in Section 3, followed by data processing and results of data analysis. The final section summarizes the finding and conclusions of the paper.

\section{Methodology}

2.1. Time-to-Collision (TTC) Calculation. TTC is defined as the time required for two vehicles to collide if they continue at their present speed and along the same path [19]. In the case of rear-end collision, Minderhoud and Bovy [20] calculate TTC as

$$
\operatorname{TTC}_{i}^{t}=\frac{\left(X_{i-1}(t)-X_{i}(t)\right)-L_{i-1}}{V_{i}(t)-V_{i-1}(t)}
$$

where $X_{i}(t)$ and $V_{i}(t)$ are the position and velocity of vehicle $i$ (the following vehicle) at $t$, respectively; $X_{i-1}(t)$ and $V_{i-1}(t)$ are the position and velocity of vehicle $i-1$ (the lead vehicle) at $t$, respectively; and $L_{i-1}$ is the length of the lead vehicle.

In the event of a lane-change conflict, when the lanechange vehicle is changing its lane, if there is another following vehicle along the original lane, there is a trajectory cross-point between the two vehicles. When the two vehicles are fast approaching, if one does not take measures, a collision will likely occur. The lane-changing conflict process is shown in Figure 1.

In situation (a), the lane-change vehicle is recorded as $A$, and the following vehicle is recorded as $B . L_{A}, V_{A}, S_{A}$ are the body length, velocity, and distance from trajectory cross-point of vehicle $A$, respectively, while $L_{B}, V_{B}, S_{B}$ are the corresponding data for vehicle $B$. We use $T_{0}$ to denote the moment of the avoidance behavior generation. Then, the travel time from the $T_{0}$ site to the trajectory cross-point, for vehicle $A$, is denoted as $T_{A}=S_{A} / V_{A}$; for vehicle $B$, it is denoted as $T_{B}=S_{B} / V_{B}$. For situation (b) and situation (c), the lane-change conflict TTC calculation method is shown in (2) and (3).

When $T_{A} \geq T_{B}$,

$$
\text { if } \begin{gathered}
T_{A} \leq T_{B}+\frac{L_{B}}{L_{B}} \\
\operatorname{TTC}_{L}=T_{A}
\end{gathered}
$$

otherwise, no conflict. 
TABLE 1: Conflicts according to the risk level, depending on the TTC value [16].

\begin{tabular}{lcc}
\hline Risk probability & TTC $(\mathrm{sec})$ & Description $\pi(E-05)$ \\
\hline 0 & $\leq 0$ & Meaningless. \\
& $>4.0$ & No conflict. \\
1 & 2.5 to 4.0 & The accident-to-conflict ratio [17] $(\pi)$ is about 0.2. \\
2 & 1.5 to 2.5 & The accident-to-conflict ratio $(\pi)$ is about 0.3. \\
3 & 1.0 to 1.5 & The accident-to-conflict ratio $(\pi)$ is about 0.6. \\
4 & $\leq 1.0$ & The accident-to-conflict ratio $(\pi)$ is above 0.8. \\
\hline
\end{tabular}

TABLE 2: Braking levels suggested by Hydén (source [18]).

\begin{tabular}{lcc}
\hline Severity grade & DRAC $\left(\mathrm{m} / \mathrm{s}^{2}\right)$ & Description \\
\hline No conflict & 0 & Evasive action not necessary \\
No conflict & 0 to 1 & Adaptation necessary \\
1 & 1 to 2 & Reaction necessary \\
2 & 2 to 4 & Considerable reaction necessary \\
3 & 4 to 6 & Heavy reaction necessary \\
4 & $\geq 6$ & Emergency reaction necessary \\
\hline
\end{tabular}

When $T_{A} \leq T_{B}$,

$$
\text { if } \begin{gathered}
T_{B} \leq T_{A}+\frac{L_{A}}{V_{A}} \\
\mathrm{TTC}_{L}=T_{B}
\end{gathered}
$$

otherwise, no conflict

Minderhoud and Bovy [20] conclude that different values are used for critical TTCs in different studies. For urban areas, Van der Horst [21] thought that a TTC of less 2.5 seconds is a critical value. However, Archer [22] takes into account that this critical value should be less than 1.5 seconds. Tageldin et al. [23] considered only traffic events with an associated minimum TTC of fewer than four seconds for evaluation. Various critical values of TTC can, therefore, be argued for. If these are translated into the minimum TTC value of conflicts, we arrive at five different conflict levels, as shown in Table 1.

\subsection{Deceleration Rate to Avoid the Crash (DRAC) Calculation.}

DRAC is defined as the required deceleration rate to avoid a collision if the offending vehicle continues with the same speed and trajectory [24]. For vehicles traveling in the same direction, DRAC can be expressed as

$$
\operatorname{DRAC}_{i, t+1}=\frac{\left(V_{i, t}-V_{i-1, t}\right)^{2}}{2\left[\left(X_{i-1, t}-X_{i, t}\right)-L_{i-1, t}\right]},
$$

where $t$ is time interval; $i$ is the following vehicle and $i-1$ is the lead vehicle; $L$ is vehicle length; and $V$ is velocity.

In the case of lane-change conflict, estimations of DRAC are obtained by

$$
\operatorname{DRAC}_{i, t+1}=\frac{V_{i, t}^{2}}{2 D_{i, t}}
$$

where $D_{i, t}$ is the distance between the projected point of collision and vehicle $i$ on the main stream. During the lanechange conflict process, if $\mathrm{TTC}_{L}=T_{A}$, then $i$ is vehicle $A$; if $\mathrm{TTC}_{L}=T_{B}$, then $i$ is vehicle $B$.

The use of DRAC allows a more intuitive (but not less arbitrary) classification of traffic conflicts [24]. Hydén [18] has suggested an alternative classification for conflicts using DRAC that is based on the expected driver reaction in order to achieve the required deceleration. The severity levels proposed are presented in Table 2.

2.3. Integrated Conflict Risk Indexes (ICRI) Calculation. At present, the conflict risk index can be divided into time indicators and an energy index. The time index reflects the risk probability of the traffic events (e.g., TTC), while the energy index reflects the severity of the traffic events (e.g., DRAC). Although TTC and DRAC are different types of conflict risk indexes, there is an inherent link between them. This inherent link is shown in (6), which is from (4).

$$
\begin{aligned}
\operatorname{DRAC}_{i, t+1} & =\frac{\left(V_{i, t}-V_{i-1, t}\right)^{2}}{2\left[\left(X_{i-1, t}-X_{i, t}\right)-L_{i-1, t}\right]} \\
& =\frac{\left(V_{i, t}-V_{i-1, t}\right)}{2\left[\left(X_{i-1, t}-X_{i, t}\right)-L_{i-1, t}\right] /\left(V_{i, t}-V_{i-1, t}\right)} \\
& =\frac{\left(V_{i, t}-V_{i-1, t}\right)}{2 \mathrm{TTC}_{i}^{t}} .
\end{aligned}
$$

From Table 2 and the relationship between TTC and DRAC in (6), we can assign a corresponding risk coefficient to each risk level, as shown in Table 3.

Because the risk level is not linear with the conflict risk index, that is, the higher the risk level, the more sensitive it is to change in the conflict risk index, we introduced the concept of odds to express the relationship between them. The risk 
TABLE 3: The risk coefficient of TTC and DRAC at different risk levels.

\begin{tabular}{lcccc}
\hline \multirow{2}{*}{ Risk level } & \multicolumn{2}{c}{ Conflict Risk Indices } & Description & Risk coefficient \\
& TTC $(\mathrm{s})$ & DRAC $\left(\mathrm{m} / \mathrm{s}^{2}\right)$ & No safety risk & 0 \\
No conflict & $\leq 0$ & 0 & No relationship between safety and indices & 0.2 \\
No conflict & $>4.0$ & 0 to 1 & The accident-to-conflict ratio is stable & 0.3 \\
1 & 2.5 to 4.0 & 1 to 2 & Low risk level & 0.6 \\
2 & 1.5 to 2.5 & 2 to 4 & Moderate risk level & 0.8 \\
3 & 1.0 to 1.5 & 4 to 6 & High risk level & \\
4 & $\leq 1.0$ & $\geq 6$ & & \\
\hline
\end{tabular}

TABLE 4: The correspondence between risk level and conflict risk index.

\begin{tabular}{lccccc}
\hline \multirow{2}{*}{ Risk level } & \multicolumn{2}{c}{ Conflict Risk Indices } & $P$ & $1-P$ & \multirow{2}{*}{$O=P / 1-P$} \\
\hline 0 & TTC $(\mathrm{s})$ & DRAC $\left(\mathrm{m} / \mathrm{s}^{2}\right)$ & 0 & 1 & 0 \\
& $\leq 0$ & 0 & 0.2 & 0.8 & 0.7 \\
1 & $>4.0$ & 1 to 1 & 0.3 & 0.4 & 0.43 \\
3 & 2.5 to 4.0 & 2 to 4 & 0.6 & 0.2 & 4.5 \\
4 & 1.5 to 2.5 & 4 to 6 & 0.8 & 0 & 0.2 \\
\hline
\end{tabular}

coefficient is similar to the probability, expressed with $P$, so $O=P / 1-P$ is the correspondence between risk level and conflict risk index, as shown in Table 4.

Referring to the literature [25] that uses the probability and impact as two independent variables to construct twodimensional rectangular coordinate system for analyzing the opportunity and threat, the Integrated Conflict Risk Index (ICRI) can be obtained by the form of Euclidean distance formula similarly. The ICRI is calculated in

$$
\operatorname{ICRI}=\sqrt{\operatorname{TTC}\left(O_{i}\right)^{2}+\operatorname{DRAC}\left(\mathrm{O}_{j}\right)^{2}}
$$

where ICRI is Integrated Conflict Risk Index; $\operatorname{TTC}\left(O_{i}\right)$ is the risk index of TTC at risk level $i, i=0,1,2,3,4$; $\operatorname{DRAC}\left(O_{j}\right)$ is the risk index of DRAC at risk level $j, j=0,1,2,3,4$.

According to Table 4 and (7), the Integrated Conflict Risk Indices matrix is constructed by TTC and DRAC, as shown in Figure 5.

We divide the risk level into four levels: zero risk (blue), low risk (green), medium risk (yellow), and high risk (red) according to the ICRI in the matrix.

2.4. Model Prototype. Lane changing is one of the main causes of traffic flow oscillation and traffic accidents. In order to study the accident mechanism caused by lane change, it is necessary to study the relationship between the risk level of lane changing and its influencing factors.

Multinomial logistic regression is a theoretical method to study the relationship between the categorical dependent variable and multiple independent variables. For a total of $k$ levels of the dependent variable, the logistic regression model at the $j$ level is shown in

$$
\ln \left[\frac{p_{j}}{1-p_{k}}\right]=\left(\beta_{j 0}+\sum_{i=1}^{n} \beta_{j i} x_{j i}\right),
$$

where $x$ is the explanatory variable; $n$ is the number of explanatory variables; and $\beta_{j 0}$ and $\beta_{j i}$ are the regression intercept and regression coefficients, respectively. From (8), the probability that a vehicle is at $j$ risk level when the vehicle executed the lane-changing maneuver is deduced as

$$
p_{j}=\frac{\exp \left(\beta_{j 0}+\sum_{i=1}^{n} \beta_{j i} x_{j i}\right)}{1+\sum_{j=1}^{k} \exp \left(\beta_{j 0}+\sum_{i=1}^{n} \beta_{j i} x_{j i}\right)},
$$

where $j=1,2,3,4$ corresponding to zero risk, low risk, medium risk, and high risk, respectively; $P_{j}$ is the probability that the lane-changing vehicle is at $j$ risk level; $x_{j 1} \sim x_{j 6}$ are the distance between the lane-changing position and the stop line, the velocity, the distance between the lane-changing vehicle (target vehicle) and the rear vehicle in the target lane (lag vehicle), the lane-change duration, the driving tendency, and the lane-change type, at $j$ risk level, respectively.

\section{Data Collection}

3.1. Questionnaire and Test. The advertisement for recruitment was posted at public locations including the Southeast University campus, Xinjiekou district transit transfer station, and some supermarkets. In addition, a web page was created (http://www.sojump.com/m/7355604.aspx?pwx=1) and people would access the website via mobile phone by using 
TABLE 5: The questionnaire for participants.

\begin{tabular}{|c|c|}
\hline Number & Questions \\
\hline \multicolumn{2}{|r|}{ Part 1 Personal Information } \\
\hline (1) & What's your gender? (A): Male; (B): Female \\
\hline (2) & When were you born? \\
\hline (3) & How many years have you been driving \\
\hline$(4)$ & What's the level of your education? \\
\hline (5) & Are you a professional driver? $\quad(\mathrm{A})$ : Yes; (B): No \\
\hline (6) & $\begin{array}{l}\text { In the last three years of driving (if you've been driving that long), have you experienced any traffic accidents? } \\
\text { (A): No; (B): Only property damaged; (C): Have had a slight injury; (D): Have had a serious injury; (E): Have caused a } \\
\text { fatality. }\end{array}$ \\
\hline \multicolumn{2}{|r|}{ Part 2 Driving Characteristics } \\
\hline (1) & Do you tend to go at more than the speed limit if there is no other vehicle interference? $\quad$ (A): Yes; (B): No \\
\hline (2) & Do you tend to rapidly accelerate or rapidly decelerate in pursuit of high speed? (A): Yes; (B): No \\
\hline (3) & Do you tend to accelerate through the dilemma zone? (A): Yes; (B): No \\
\hline$(4)$ & Do you tend to closely follow the front vehicle? (A): Yes; (B): No \\
\hline$(5)$ & Do you tend to overtake the front vehicle if there is only a small space between you? $\quad$ (A): Yes; (B): No \\
\hline (6) & Do you tend to turn on your indicator before you start to change lanes? $\quad$ (A): Yes; (B): No \\
\hline (7) & $\begin{array}{l}\text { When the adjacent lanes have parallel vehicles, you } \\
\text { (A): Tend to accelerate; (B): Tend to decelerate; (C): Do not respond }\end{array}$ \\
\hline (8) & $\begin{array}{l}\text { If you need to turn or make a U-turn at the next intersection, you } \\
\text { (A): Give priority to reaching the intersection as soon as possible and then inserting yourself into the target lane; (B): Give } \\
\text { priority to changing to the target lane even if you need to queue }\end{array}$ \\
\hline (9) & You overtake at the curve $\quad$ (A): Constantly; (B): Occasionally; (C): Never \\
\hline$(10)$ & $\begin{array}{l}\text { When you are turning right, you _--_- avoid non-motor vehicles or pedestrians } \\
\text { (A): Generally do not; (B): Occasionally; (C): Usually; (D): Always }\end{array}$ \\
\hline (11) & Do you tend to be irritable when you are waiting for a green light or in traffic jams? $\quad$ (A): Yes; (B): No \\
\hline$(12)$ & When other drivers commit operating errors, you tend toward $\quad(A)$ : Anger; (B): Forgiveness \\
\hline (13) & $\begin{array}{l}\text { When the front vehicle is moving at a low speed in the current lane but you can't change lanes, you tend to } \\
\text { (A): Honk or flash; (B): Just follow }\end{array}$ \\
\hline$(14)$ & $\begin{array}{l}\text { Near the intersection, you suddenly realize that you are in the wrong lane. You tend to } \\
\text { (A): Brake to change to the lane you want; (B): Make a U-turn at the next intersection }\end{array}$ \\
\hline$(15)$ & $\begin{array}{l}\text { When parking spaces are limited and there is only one parking space closer to the other side between you and the } \\
\text { oncoming vehicle, you tend to } \\
\text { (A): Accelerate to occupy the parking space; (B): Look for another parking space }\end{array}$ \\
\hline
\end{tabular}

WeChat scanning QR code. Respondents could complete the questionnaire and submit answer sheets online. The sample of target drivers was selected considering several impact factors such as gender, age, driving experience, and educational background, and then a sample consisting of one hundred and thirteen participants was fixed for experimental purposes. It can be considered that the sample can reflect the distribution of the general population of drivers, and the analyzed data is representative. In order to classify driving tendencies, each participant needed to undergo a number of tests as well as fill out a questionnaire.

As shown in Table 5, the questionnaire has two parts: the first part concerns basic personal information about the participants, including gender, age, driving experience, and educational qualifications (the contribution of personal attribute of the participants is shown in Figure 2); the second part is about the driving characteristics of participants in different environmental conditions, including the level of subjective safety cognition, aggressiveness, and driving skills. We tested the reliability and validity of the second part of the questionnaire. For reliability, Cronbach's Alpha is 0.854; for validity, we calculated the Pearson correlation coefficient between the scores of each question and the total scores, and $90 \%$ of these questions and the total scores have a correlation at 0.01 and 0.05 significance levels. The questionnaire has good reliability and validity and can be used for investigation.

The tests are also divided into two parts: the first part is the static test (flash testing), including a response time test and a speed estimation test; the second part is the dynamic test (real vehicle testing), including a braking frequency test, a driving force frequency test, and a changing lane frequency test.

For the response time test, we designed a flash test. The test repeats ten times. Each time, one of the four direction (up, down, left, and right) arrows randomly appeared on the screen. When the participant saw the arrow, he/she had to 


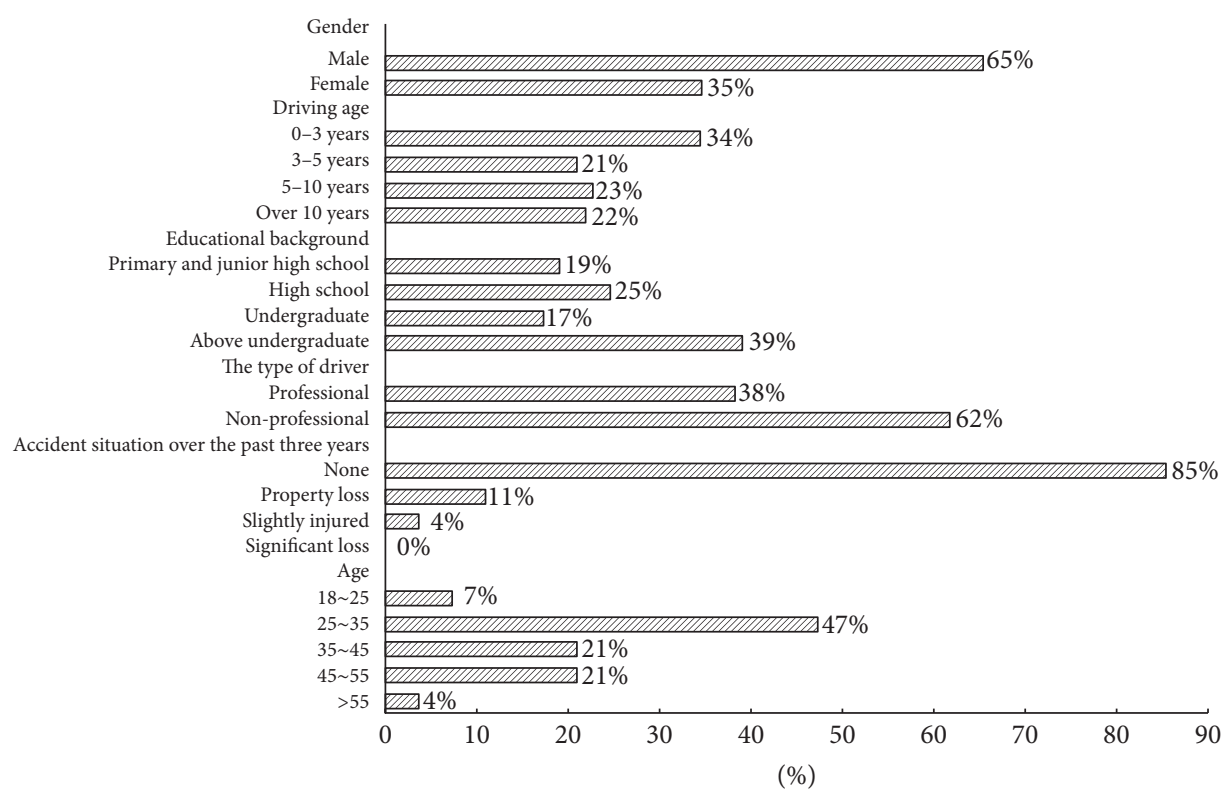

FIGURE 2: The contribution of personal attribute of the participants.

press the corresponding arrow on the keyboard as soon as possible. Pressing any key would start the next test. Every time, the outcome (correct or incorrect) and the response time would be displayed on the screen. At the end, all the results were saved in a txt document.

For the speed estimation test, we also designed a flash test. The test repeats five times. When the test starts, a little ball appears on the left side of the screen and moves at uniform speed to the right side. On the right side of the screen, there is a black area in which there is a vertical red dotted line. When the little ball moves into the black area, it is not visible. The participant has to press any key when he/she estimates that the little ball has reached the vertical red dotted line based on the speed of the ball. The real time and the estimated time of the participant will show on the screen. Each time, the speed of the little ball and the location of the vertical red dotted line are random. At the end, all the results were saved in a txt document.

For the dynamic test, we used a car equipped with a driving recorder to collect data. The data collection route is about $1.8 \mathrm{~km}$, in Nanjing Xuanwu District, and contains six signal lights. Two driving recorders were installed in the instrumented car, one on the front windscreen to record lanechanging times and the other with a LED light near the brake of the car to collect the times of braking and driving force. Each participant drove the instrumented car along the data collection route twice, and the driving time was recorded. After the test, the braking frequency, driving force frequency, and lane-changing frequency of each participant could be calculated.

3.2. The Experiment. The questionnaire and tests described above were followed by the experiment, which lasted from January to the end of March 2016. The purpose of this experiment was to collect the trajectory data of different driving tendencies of participants while implementing lanechanging maneuvers when approaching an intersection. We chose two urban signalized intersections as the experimental observation points. For each point, one camera was set up on top of a roadside building to cover a studied approach. The first studied approach is a northbound approach along TaiPing North Avenue ( $\approx 220 \mathrm{~m}$ ); the second studied approach is an eastbound approach along HeXi Avenue $(\approx 280 \mathrm{~m})$. For each studied approach, over 150 hours of recorded video data were used to capture lane-changing maneuvers.

We designed the experimental route to generate lanechanging behavior on the studied approach. If the instrumented car turns right/left at an intersection, the lanechanging behavior in the studied approach is objectivedriven lane changing, meaning that the driver must change lanes prior to turning at the next signal. If the instrumented car goes straight through the intersection, the lane-changing behavior in the studied approach is efficiency-driven lane changing, which is pursued for a better driving situation. Each participant drove our instrumented car along the experimental route until the instrumented car passed through each selected intersection approach nine times, being three times turning right, three times driving straight, and three times turning left. We put a sign on the roof of our instrumented car so that it was easy to distinguish from other vehicles in the video. As shown in Figure 3, we recorded the experimental process at one of our studied approaches.

The video for each intersection approach was recorded from 8:00 a.m. to noon and 1:00 p.m. to 5:00 p.m. Each participant spent about two hours on this experiment, including the questionnaire and tests mentioned above. We ran the driving recorder and camera synchronously. An experimenter sat in the passenger seat of the instrumented car to record the time 
TABLE 6: The descriptive statistics of the questionnaire survey and tests for participants.

\begin{tabular}{|c|c|c|c|c|c|}
\hline Items & $N$ & Min & Max & Mean & STD \\
\hline Questionnaire scores & 113 & 6.000 & 32.000 & 22.717 & 7.073 \\
\hline Response time accuracy & 113 & 0.900 & 1.000 & 0.975 & 0.043 \\
\hline Response time & 113 & 0.440 & 1.360 & 0.686 & 0.187 \\
\hline Speed estimation time error & 113 & 0.060 & 0.680 & 0.324 & 0.137 \\
\hline Driving force frequency & 113 & 2.550 & 5.500 & 3.544 & 0.730 \\
\hline Braking frequency & 113 & 2.490 & 5.880 & 3.812 & 0.958 \\
\hline Lane-changing frequency & 113 & 0.210 & 0.950 & 0.556 & 0.159 \\
\hline
\end{tabular}
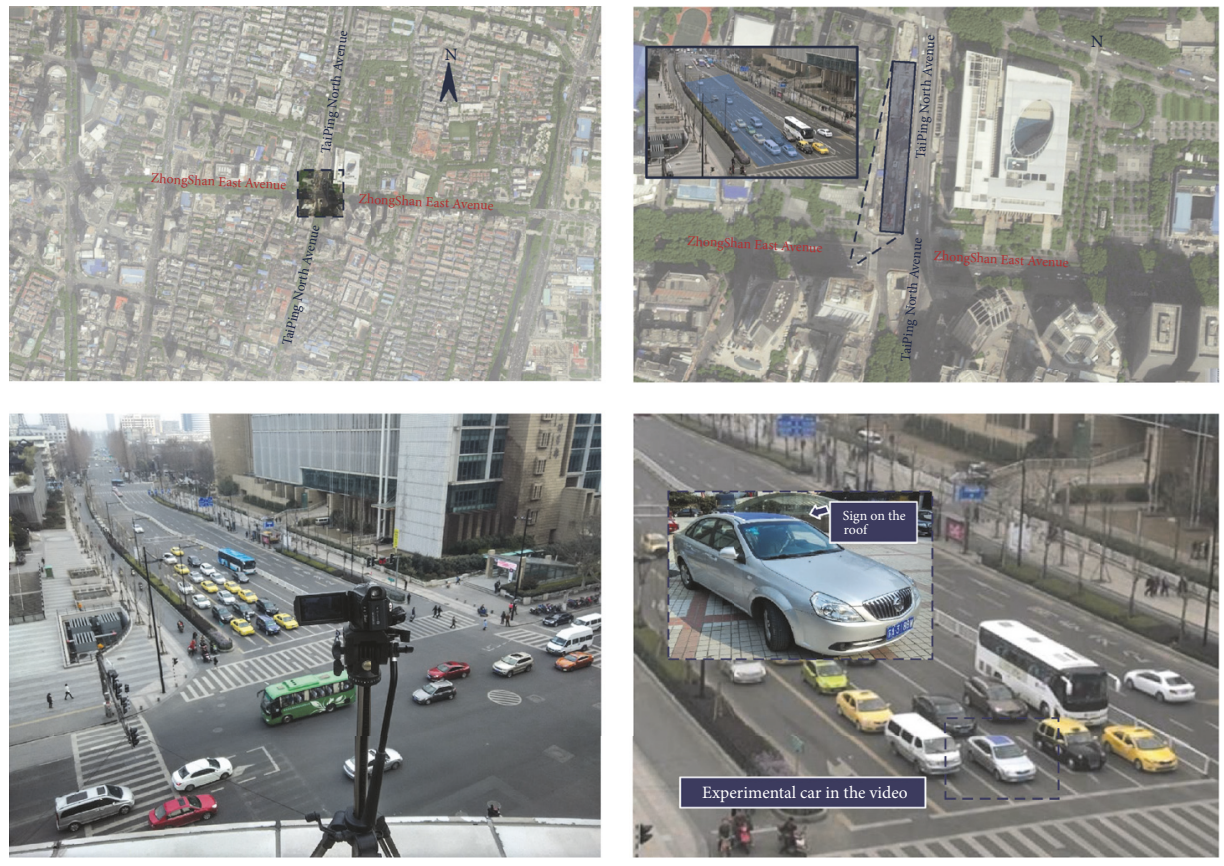

FIGURE 3: The recording of the experimental process.

of lane changing so that we could easily find the instrumented car in the video and extract the trajectory data.

\section{Data Processing}

4.1. Driving Tendency Discrimination. The statistical results of the questionnaire survey and tests are shown in Table 6.

The original data were normalized, and driving tendencies were clustered into three categories by $K$-means, as shown in Tables 7 and 8.

Twenty-three participants belong to the "aggressive" type, eighteen participants belong to the "conservative" type, and seventy-two participants belong to the "steady" type according to the algorithm of $K$-means.

4.2. Trajectory Data Extraction. In this section, the extraction method of trajectory data involves two steps. First, the Tracker Video Analysis and Modeling Tool was used to extract the coordinates of the vehicles in the video. Secondly, the imaging principle was used to match the coordinates in the video with the location in the real world.

Tracker [26] is a free video analysis and modeling tool built on the Open Source Physics (OSP) Java framework. It has manual and automated object tracking with position, velocity, and acceleration overlays and data. In this paper, Tracker was used for tracking and extracting the coordinates of the vehicles. First, we needed to put the video into Tracker and then build a reference frame. Track points were set up on the right corner of the front windscreen, and the sampling frequency was set to five frames per second. As shown in Figure 4(a), Tracker can generate real-time coordinates and space-time diagrams of subject vehicles while putting out three columns of data-time $(t), x$ coordinate, and $y$ coordinate-to record the coordinates of the subject vehicles.

The purpose of utilizing this imaging principle was to transfer the coordinates from two-dimensional video to their three-dimensional location in the real world. Velocity can be calculated by the direction and distance of the subject vehicle between contiguous data. Acceleration can be calculated by 
TABLE 7: Final cluster centers and ANOVA.

\begin{tabular}{|c|c|c|c|c|c|c|c|c|c|}
\hline \multirow{2}{*}{$\begin{array}{l}\text { Normalized items } \\
Z \text { score }\end{array}$} & \multicolumn{3}{|c|}{ Clustering center } & \multicolumn{2}{|l|}{ Cluster } & \multicolumn{2}{|l|}{ Error } & \multirow{2}{*}{$F$} & \multirow{2}{*}{ Sig. } \\
\hline & 1 & 2 & 3 & Mean square & $\mathrm{df}$ & Mean square & $\mathrm{df}$ & & \\
\hline Questionnaire scores & -0.384 & 0.015 & 0.433 & 3.390 & 2 & .957 & 110 & 3.544 & .032 \\
\hline Response time accuracy & 0.571 & 0.123 & -1.222 & 17.742 & 2 & .696 & 110 & 25.507 & .000 \\
\hline Response time & 0.261 & -0.482 & 1.595 & 32.055 & 2 & .435 & 110 & 73.626 & .000 \\
\hline Speed estimation time error & 0.671 & -0.324 & 0.440 & 10.710 & 2 & .823 & 110 & 13.006 & .000 \\
\hline Driving force frequency & 1.679 & -0.339 & -0.790 & 42.151 & 2 & .252 & 110 & 167.399 & .000 \\
\hline Braking frequency & 1.527 & -0.380 & -0.431 & 33.668 & 2 & .406 & 110 & 82.917 & .000 \\
\hline Lane-changing frequency & 0.790 & -0.094 & -0.634 & 11.105 & 2 & .816 & 110 & 13.604 & .000 \\
\hline
\end{tabular}

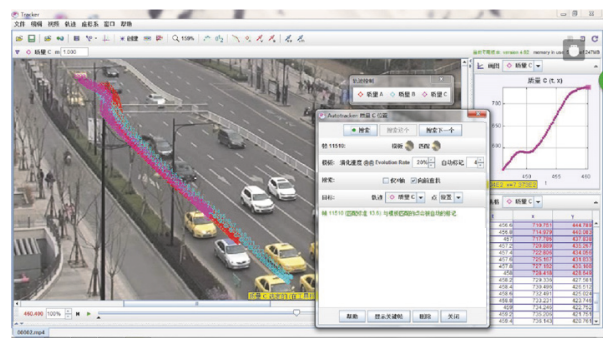

(a)

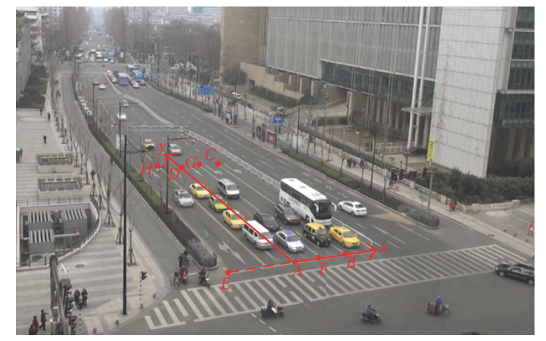

(b)

FIGURE 4: The extraction method of trajectory data.

TABLE 8: Distance between Final cluster centers and number of cases in each cluster.

\begin{tabular}{lcccc}
\hline Cluster & 1 & 2 & 3 & The number of cases in each cluster \\
\hline Cluster & & & & \\
1 & & 2.717 & 4.204 & \\
2 & 3.223 & & 3.223 & \\
3 & 4.204 & 3.223 & & 18 \\
Effective & & & 113 & 72 \\
Missing & & & 0 & \\
\hline
\end{tabular}

the speed difference in sampling intervals. According to the imaging principle, there is a fixed matrix transformation between the object space coordinate system and image plane coordinates. The relationship is as follows:

$$
\begin{gathered}
X_{s}=\frac{C_{1}+C_{2} X_{r}+C_{3} Y_{r}+C_{4} Z_{r}}{1+C_{9} X_{r}+C_{10} Y_{r}+C_{11} Z_{r}}, \\
Y_{s}=\frac{C_{5}+C_{6} X_{r}+C_{7} Y_{r}+C_{8} Z_{r}}{1+C_{9} X_{r}+C_{10} Y_{r}+C_{11} Z_{r}},
\end{gathered}
$$

where $X_{s}$ and $Y_{s}$ are, respectively, the $x$ coordinate and $y$ coordinate of the target object in the image, and $X_{r}, Y_{r}$, and $Z_{r}$ are, respectively, the $x$ coordinate, $y$ coordinate, and $z$ coordinate (vertical coordinates) in the real world. $C_{1}, C_{2}$, $C_{3}, C_{4}, C_{5}, C_{6}, C_{7}, C_{8}, C_{9}, C_{10}$, and $C_{11}$ are constant

\begin{tabular}{|c|c|c|c|c|c|c|}
\hline & \multicolumn{5}{|c|}{ DRAC (severity grade) } \\
\hline & & 0 & 1 & 2 & 3 & 4 \\
\hline \multirow{5}{*}{ 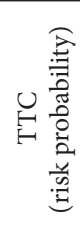 } & 0 & 0 & 0.250 & 0.430 & 1.500 & 4.000 \\
\hline & 1 & 0.25 & 0.354 & 0.497 & 1.521 & 4.008 \\
\hline & 2 & 0.43 & 0.497 & 0.608 & 1.560 & 4.023 \\
\hline & 3 & 1.5 & 1.521 & 1.560 & 2.121 & 4.272 \\
\hline & 4 & 4.0 & 4.008 & 4.023 & 4.272 & 5.657 \\
\hline
\end{tabular}
coefficients.
FIgURE 5: The Integrated Conflict Risk Indices matrix.

In this paper, $Z_{r}$ is considered a constant coefficient because of the negligible road slope grade. The equation above can, therefore, be simplified to

$$
\begin{aligned}
& X_{r}=\frac{C_{1}+C_{2} X_{s}+C_{3} Y_{s}}{1+C_{7} X_{s}+C_{8} Y_{s}}, \\
& Y_{r}=\frac{C_{4}+C_{5} X_{s}+C_{6} Y_{s}}{1+C_{7} X_{s}+C_{8} Y_{s}} .
\end{aligned}
$$

We established a coordinate system at the intersection approach by selecting four noncollinear points and measuring their actual coordinates. Then we had to get the image coordinates in the video from Tracker and put these eight sets of data into (11), so we could calibrate the eight constant coefficients. As depicted in Figure 4(b), we selected eight feature points, where $B, C, E$, and $G$ are the coordinate points and $A, D, F$, and $H$ are the error-checking points.

A total of 686 trajectory data were extracted. The categorical variables were encoded, as shown in Figure 5, and the continuous variables were counted, as shown in Table 9. 
TABLE 9: Descriptive statistics for categorical variables.

\begin{tabular}{|c|c|c|c|}
\hline Categorical variables & Code & Frequency & Percentage \\
\hline \multirow{4}{*}{$\begin{array}{l}\text { Risk level } \\
\text { (ICRI) }\end{array}$} & 1: zero risk level & 314 & $45.8 \%$ \\
\hline & 2: low risk level & 197 & $28.7 \%$ \\
\hline & 3: medium risk level & 78 & $11.4 \%$ \\
\hline & 4: high risk level & 97 & $14.1 \%$ \\
\hline \multirow{3}{*}{$\begin{array}{l}\text { Driving tendency } \\
\left(X_{5}\right)\end{array}$} & 1: aggressive tendency & 240 & $35.0 \%$ \\
\hline & 2: conservative tendency & 131 & $19.1 \%$ \\
\hline & 3: steady tendency & 315 & $45.9 \%$ \\
\hline \multirow{2}{*}{$\begin{array}{l}\text { Lane-changing type } \\
\left(X_{6}\right)\end{array}$} & 0: efficiency & 318 & $46.4 \%$ \\
\hline & 1: objective & 368 & $53.6 \%$ \\
\hline
\end{tabular}

TABLE 10: Descriptive statistics for continuous variables.

\begin{tabular}{|c|c|c|c|c|c|}
\hline Continuous variables & $N$ & Min & Max & Mean & Std. deviation \\
\hline \multicolumn{6}{|l|}{$X_{1}$} \\
\hline Distance from stop line & 686 & 18.490 & 259.410 & 103.779 & 38.948 \\
\hline \multicolumn{6}{|l|}{$X_{2}$} \\
\hline Velocity & 686 & 0.620 & 19.690 & 8.103 & 3.296 \\
\hline \multicolumn{6}{|l|}{$X_{3}$} \\
\hline Distance from lag vehicle & 686 & 0.360 & 71.560 & 11.750 & 10.349 \\
\hline \multicolumn{6}{|l|}{$X_{4}$} \\
\hline Lane-changing duration & 686 & 0.940 & 17.000 & 4.637 & 1.986 \\
\hline Effective $N$ & 686 & & & & \\
\hline
\end{tabular}

TABLE 11: Model fitting information.

\begin{tabular}{|c|c|c|c|c|}
\hline \multirow{2}{*}{ Model } & Model fitting criteria & \multicolumn{3}{|c|}{ Likelihood ratio tests } \\
\hline & -2 Log Likelihood & Chi-Square & $\mathrm{df}$ & Sig. \\
\hline Intercept only & 1701.023 & \multirow{2}{*}{549.217} & \multirow{2}{*}{21} & \multirow{2}{*}{.000} \\
\hline Final & 1151.805 & & & \\
\hline
\end{tabular}

\section{Results of Data Analysis}

5.1. Model Calibration. The variables in Tables 9 and 10 were substituted into (8), and the model parameters were calibrated with zero risk level as a reference standard. The Model Fitting Information, Likelihood Ratio Tests, and Parameter Estimates are shown in Tables 11, 12, and 13, respectively.

5.2. Analysis of Results. Table 11 shows that the final results are significantly different from the model with only intercepts, indicating that the model has the ability to make accurate predictions. Cox and Snell, Nagelkerke, and McFadden are $.551, .601$, and .323 , respectively, indicating that the model is well fitted.

As can be seen from Table 12, velocity, the distance from lag vehicle, and the driving tendency are significant at the $P<0.05$, indicating that these three factors for the risk level of lane-changing execution have a significant impact. On the contrary, the distance from stop line, lane-changing duration, and lane-changing type have no significant impact on the risk level of lane-changing execution. For the parameter variable of $P \leq 0.05$, it has not been removed; the purpose is to combine multiple independent variables nonparametric test analysis, so that the results can reflect the process of risk estimation model more directly and thereby improve its fit.

Table 13 shows the relevant data for parameter estimation. The reference group of the categorical dependent variable is risk level 1 (zero risk level). For the categorical independent variables, the "steady" type and the "objective" type are the reference groups of the driving tendency variable and of the lane-changing type variable, respectively. The significant level (Sig.) represents the reliability of the independent variable parameter to the interpretation of the dependent variable. When $P \leq 0.05$, the level of the reliability of the independent variable parameter for the interpretation of the dependent variable is greater than or equal to $95 \%$.

As shown in Table 13, the distances from lag vehicle (the distance between the target vehicle and the lag vehicle) at risk level 2 (low risk level), risk level 3 (medium risk level), and risk level 4 (high risk level) are significantly different from 
TABLE 12: Likelihood ratio tests.

\begin{tabular}{|c|c|c|c|c|}
\hline \multirow{2}{*}{ Effect } & \multirow{2}{*}{$\begin{array}{c}\text { Model fitting criteria } \\
-2 \log \text { Likelihood of reduced model }\end{array}$} & \multicolumn{3}{|c|}{ Likelihood ratio test } \\
\hline & & Chi-Square & df & Sig. \\
\hline Intercept & 1151.805 & .000 & 0 & . \\
\hline$X 1$ & 1156.041 & 4.236 & 3 & .237 \\
\hline$X 2$ & 1163.428 & 11.623 & 3 & .009 \\
\hline$X 3$ & 1603.649 & 451.844 & 3 & .000 \\
\hline$X 4$ & 1153.970 & 2.165 & 3 & .539 \\
\hline$X 5$ & 1176.139 & 24.334 & 6 & .000 \\
\hline$X 6$ & 1153.275 & 1.470 & 3 & .689 \\
\hline
\end{tabular}

that at the risk level 1 . When it reduces $1 \mathrm{~m}$, the odds of the risk of collisions caused by the lane-changing at risk level 2 , risk level 3, and risk level 4 are increased by $0.937,0.524$, and 0.299 , respectively. This shows that if the other factors are under the same conditions, the shorter the distance from lag vehicle, the higher the risk of conflict during lanechanging execution. Results relating to aggressive driving tendency are very similar to the case of the distances from lag vehicle. However, the difference between "steady" type and "conservative" type is not significant.

The velocity at all risk levels except risk level 2 has a significant difference compared to that at the risk level 1. When it increased $1 \mathrm{~m} / \mathrm{s}$, the odds of the risk of collisions caused by the lane-changing at risk level 3 and risk level 4 increase by 0.197 and 0.159 , respectively. The insignificance at risk level 2 means that the velocity is statistically insignificant for triggering low risk level which more depends on the distance from lag vehicle and whether the driver belongs to "aggressive" type.

It is noteworthy that, for the distance from stop line, the significance can appear at risk level 4 only. This also explains the intuitive feelings that in "objective" type lane-changing, the shorter the distance from stop line, the higher the risk of conflict during lane-changing execution.

5.3. Application Examples. Assuming that the lane-changing maneuver is implemented at the rate of $18 \mathrm{~m} / \mathrm{s}$ at $80 \mathrm{~m}$ from the stop line, the distance from the lag vehicle is $2 \mathrm{~m}$, the lanechanging duration is four seconds, the tendency of the driver is aggressive, and the type of lane-changing is objectivedriven lane changing, the probability of the risk level of the vehicle during the lane change can be found.

According to (8), the effect value under low risk, medium risk, and high risk during the implementation of lanechanging was respectively calculated by using the parameter calibration result of Table 13 .

$$
\begin{aligned}
\ln \frac{P_{2}}{P_{1}}= & 0.706-0.003 \times 80-0.013 \times 18-0.065 \times 2 \\
& \quad-0.097 \times 4+1.079=0.793, \\
\ln \frac{P_{3}}{P_{1}}= & 1.770-0.006 \times 80+0.197 \times 18-0.627 \times 2 \\
& \quad-0.075 \times 4+2.089=5.371,
\end{aligned}
$$

$$
\begin{aligned}
\ln \frac{P_{4}}{P_{1}}= & 4.537-0.011 \times 80+0.159 \times 18-1.158 \times 2 \\
& -0.015 \times 4+2.177=6.32,
\end{aligned}
$$

where $P_{2}, P_{3}, P_{4}$ are the probability of low risk, medium risk, and high risk, respectively. (9):

The probability of risk level was respectively calculated by

$$
\begin{aligned}
& P_{2}=\frac{e^{0.793}}{1+e^{0.793}+e^{5.371}+e^{6.32}}=0.003, \\
& P_{3}=\frac{e^{5.371}}{1+e^{0.793}+e^{5.371}+e^{6.32}}=0.278, \\
& P_{4}=\frac{e^{6.32}}{1+e^{0.793}+e^{5.371}+e^{6.32}}=0.718, \\
& P_{1}=1-0.003-0.278-0.718=0.001
\end{aligned}
$$

It can be seen that, in this case, the probability of the vehicle being at zero risk, low risk, medium risk, and high risk is $0.001,0.003,0.278$, and 0.718 , respectively. If we changed the tendency of the driver from "aggressive" to "conservative," the corresponding value becomes $0.001,0.020,0.601$, and 0.378, respectively. According to Two Independent Samples Test, it was found that driving tendency has a significant effect on the risk of collisions caused by the lane-changing. The result means that, compared with the existing lane-change model [14], adding the factor of driving tendency will improve the accuracy of crash risk prediction model of lane-change.

\section{Summary and Conclusions}

A traffic conflict risk prediction model with a new index is developed in this study. The major significance of this study is summarized as follows. Firstly, the time-to-collision (TTC) value and deceleration rate to avoid the crash (DRAC) value were calculated based on the data extracted from the video and Tracker, respectively. The measurement accuracy of both these indices was significantly improved. Based on these two indices, an ICRI including risk probability and severity is proposed, and the safety status of vehicle operation can be evaluated by this index. Secondly, based on the results of a questionnaire survey and related tests, the $K$-mean clustering 
TABle 13: Parameter estimates.

\begin{tabular}{|c|c|c|c|c|c|c|c|c|c|}
\hline \multirow[t]{2}{*}{ Risk level $^{\mathrm{a}}$} & \multirow[t]{2}{*}{ Variables } & \multirow[t]{2}{*}{$B$} & \multirow[t]{2}{*}{$\begin{array}{l}\text { Std. } \\
\text { Err. }\end{array}$} & \multirow[t]{2}{*}{ Wald } & \multirow[t]{2}{*}{$\mathrm{df}$} & \multirow[t]{2}{*}{ Sig. } & \multirow[t]{2}{*}{$\operatorname{Exp}(B)$} & \multicolumn{2}{|c|}{$\begin{array}{l}\text { 95\% confidence interval for Exp } \\
(B)\end{array}$} \\
\hline & & & & & & & & Lower bound & Upper bound \\
\hline \multirow{12}{*}{2} & Intercept & .706 & .705 & 1.003 & 1 & .317 & & & \\
\hline & $X 1$ & -.003 & .003 & .631 & 1 & .427 & .997 & .991 & 1.004 \\
\hline & $X 2$ & -.013 & .040 & .100 & 1 & .752 & .987 & .913 & 1.068 \\
\hline & $X 3$ & -.065 & .013 & 26.615 & 1 & .000 & .937 & .914 & .960 \\
\hline & $X 4$ & -.097 & .069 & 1.937 & 1 & .164 & .908 & .792 & 1.040 \\
\hline & $X 5$ & & & & & & & & \\
\hline & 1 & 1.079 & .376 & 8.225 & 1 & .004 & 2.942 & 1.407 & 6.150 \\
\hline & 2 & .507 & .394 & 1.653 & 1 & .199 & 1.660 & .767 & 3.593 \\
\hline & 3 & $0^{\mathrm{b}}$ & . & & 0 & . & . & . & . \\
\hline & $X 6$ & & & & & & & & \\
\hline & 0 & .105 & .221 & .226 & 1 & .634 & 1.111 & .720 & 1.714 \\
\hline & 1 & $0^{\mathrm{b}}$ & . & . & 0 & . & . & . & . \\
\hline \multirow{12}{*}{3} & Intercept & 1.770 & 1.265 & 1.957 & 1 & .162 & & & \\
\hline & $X 1$ & -.006 & .005 & 1.228 & 1 & .268 & .994 & .984 & 1.004 \\
\hline & $X 2$ & .197 & .065 & 9.147 & 1 & .002 & 1.217 & 1.072 & 1.383 \\
\hline & $X 3$ & -.627 & .075 & 70.428 & 1 & .000 & .534 & .461 & .618 \\
\hline & $X 4$ & -.075 & .127 & .350 & 1 & .554 & .928 & .724 & 1.189 \\
\hline & $X 5$ & & & & & & & & \\
\hline & 1 & 2.089 & .657 & 10.114 & 1 & .001 & 8.075 & 2.229 & 29.256 \\
\hline & 2 & .363 & .718 & .256 & 1 & .613 & 1.438 & .352 & 5.868 \\
\hline & 3 & $0^{\mathrm{b}}$ & . & & 0 & . & . & . & . \\
\hline & $X 6$ & & & & & & & & \\
\hline & 0 & -.210 & .379 & .306 & 1 & .580 & .811 & .386 & 1.705 \\
\hline & 1 & $0^{\mathrm{b}}$ & . & . & 0 & . & . & . & . \\
\hline \multirow{12}{*}{4} & Intercept & 4.537 & 1.354 & 11.232 & 1 & .001 & & & \\
\hline & $X 1$ & -.011 & .006 & 4.122 & 1 & .042 & .989 & .978 & 1.000 \\
\hline & $X 2$ & .159 & .073 & 4.723 & 1 & .030 & 1.172 & 1.016 & 1.352 \\
\hline & $X 3$ & -1.158 & .117 & 97.240 & 1 & .000 & .314 & .250 & .395 \\
\hline & $X 4$ & -.015 & .153 & .010 & 1 & .922 & .985 & .730 & 1.330 \\
\hline & $X 5$ & & & & & & & & \\
\hline & 1 & 2.177 & .733 & 8.831 & 1 & .003 & 8.820 & 2.098 & 37.069 \\
\hline & 2 & -.960 & .865 & 1.233 & 1 & .267 & .383 & .070 & 2.085 \\
\hline & 3 & $0^{\mathrm{b}}$ & . & . & 0 & . & . & . & 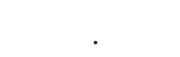 \\
\hline & $X 6$ & & & & & & & & \\
\hline & 0 & -.387 & .414 & .874 & 1 & .350 & .679 & .302 & 1.528 \\
\hline & 1 & $0^{\mathrm{b}}$ & . & . & 0 & . & . & . & . \\
\hline
\end{tabular}

Note. ${ }^{a}$ The reference category is $1 ;{ }^{b}$ this parameter is set to zero because it is redundant.

analysis method was used to classify the driving tendency of the participants, the trajectory data of different driving tendency were extracted, and the driving tendency was taken as an influential factor for the safety of the lane-changing. Finally, a traffic conflict risk prediction model is proposed to predict the security risk of lane-changing. As a result, a similar method can be applied to the approach of urban intersections or merging areas in the interchange.
Detailed data sets from experimental data, including questionnaire, response time test, speed estimation test, braking frequency test, driving force frequency test, and changing lane frequency test, and from field data, including the distance from stop line, the vehicle velocity, the distance from lag vehicle, lane-change duration, driving tendency, and lane-changing type, are adopted in the study. Some important findings are summarized in the following statements: 
(1) Response time and speed estimation capability of drivers have no significant effect on driving tendency. On the contrary, braking frequency, driving force frequency, and changing lane frequency have a significant effect.

(2) The vehicle velocity, distance from the lag, and driving tendency have a significant effect on the risk level of lane-changing execution. On the contrary, the distance from stop line, lane-change duration, and lane-changing maneuvers type have no significant impact on the risk level of lane-changing execution.

(3) This paper reports the explorative effort of developing a new traffic conflict risk prediction model using ICRI as an evaluation index. At the end of the article, the model is used to analyze an application example, and the probability of various risks under certain conditions is obtained. The results of the study can provide the basis for conflict risk in the existing lane-changing models. Such a study offers significant potential for engineering applications and safety evaluation.

\section{Conflicts of Interest}

The authors declare that they have no conflicts of interest.

\section{Acknowledgments}

This research was funded by the National Natural Science Foundation of China (Project no. 51478110).

\section{References}

[1] C. Böffel and J. Müsseler, "Adjust your view! Wing-mirror settings influence distance estimations and lane-change decisions," Transportation Research Part F: Traffic Psychology and Behaviour, vol. 35, pp. 112-118, 2015.

[2] T. Petzoldt, N. Bär, C. Ihle, and J. F. Krems, "Learning effects in the lane change task (LCT) - Evidence from two experimental studies," Transportation Research Part F: Traffic Psychology and Behaviour, vol. 14, no. 1, pp. 1-12, 2011.

[3] P. Hofmann, G. Rinkenauer, and D. Gude, "Preparing lane changes while driving in a fixed-base simulator: Effects of advance information about direction and amplitude on reaction time and steering kinematics," Transportation Research Part F: Traffic Psychology and Behaviour, vol. 13, no. 4, pp. 255-268, 2010.

[4] S. Moridpour, G. Rose, and M. Sarvi, "Effect of surrounding traffic characteristics on lane changing behavior," Journal of Transportation Engineering, vol. 136, no. 11, pp. 973-985, 2010.

[5] P. Hidas, "Modelling vehicle interactions in microscopic simulation of merging and weaving," Transportation Research Part C: Emerging Technologies, vol. 13, no. 1, pp. 37-62, 2005.

[6] L. Leclercq, N. Chiabaut, J. Laval, and C. Buisson, "Relaxation phenomenon after lane changing experimental validation with NGSIM data set," Transportation Research Record, no. 1999, pp. 79-85, 2007.

[7] G. Bifulco, F. Galante, L. Pariota, M. Russo Spena, and P. Del Gais, "Data collection for traffic and drivers' behaviour studies: a large-scale survey," Procedia - Social and Behavioral Sciences, vol. 111, pp. 721-730, 2014.

[8] P. Hidas, "Modelling lane changing and merging in microscopic traffic simulation," Transportation Research Part C: Emerging Technologies, vol. 10, no. 5-6, pp. 351-371, 2002.

[9] P. Hidas and P. Wagner, "Review of data collection methods for microscopic traffic simulation," in Proceedings of the 10th World Conference on Transport Research, Istanbul , Turkey, 2004, Review of data collection methods for microscopic traffic simulation[C].

[10] Z. Zheng, "Recent developments and research needs in modeling lane changing," Transportation Research Part B: Methodological, vol. 60, pp. 16-32, 2014.

[11] C. Hill, L. Elefteriadou, and A. Kondyli, "Exploratory analysis of lane changing on freeways based on driver behavior," in Proceedings of the Transportation Research Board 92nd Annual Meeting, Washington, DC, USA, 2013.

[12] S. Moridpour, M. Sarvi, and G. Rose, "Modeling the lanechanging execution of multiclass vehicles under heavy traffic conditions," Transportation Research Record, no. 2161, pp. 11-19, 2010.

[13] S. Moridpour, M. Sarvi, and G. Rose, "Lane changing models: a critical review," Transportation Letters, vol. 2, no. 3, pp. 157-173, 2010.

[14] D. Sun and L. Elefteriadou, "Research and implementation of lane-changing model based on driver behavior," Transportation Research Record, no. 2161, pp. 1-10, 2010.

[15] G. D. Roman, D. Poulter, E. Barker, F. P. McKenna, and R. Rowe, "Novice drivers' individual trajectories of driver behavior over the first three years of driving," Accident Analysis and Prevention, vol. 82, pp. 61-69, 2015.

[16] Y. Guo, Signalized Intersection Safety Evaluation Techniques Based on Traffic Conflict Theory, Southeast University, 2016.

[17] E. Hauer and P. Garder, "Research into the validity of the traffic conflicts technique," Accident Analysis and Prevention, vol. 18, no. 6, pp. 471-481, 1986.

[18] C. Hydén, “Traffic safety work with video-processing," Tech. Rep., Transportation Department, University Kaiserslautern, 1996.

[19] J. Hayward, Near misses as a measure of safety at urban intersections, Pennsylvania Transportation and Traffic Safety Center, 1971.

[20] M. M. Minderhoud and P. H. L. Bovy, "Extended time-tocollision measures for road traffic safety assessment," Accident Analysis and Prevention, vol. 33, no. 1, pp. 89-97, 2001.

[21] A. R. A. Van der Horst, A time based analysis of road user behaviour in normal and critical encounters.

[22] J. Archer, Indicators for Traffic Safety Assessment And Prediction and Their Application in Micro-Simulation Modelling: A Study of Urban and Suburban Intersections, KTH, Stockholm, Sweden, 2005.

[23] A. Tageldin, T. Sayed, K. Shaaban, and M. H. Zaki, "Automated analysis and validation of right-turn merging behavior," Journal of Transportation Safety and Security, vol. 7, no. 2, pp. 138-152, 2015.

[24] F. Cunto, Assessing safety performance of transportation systems using microscopic simulation.

[25] D. Hillson, "Extending the risk process to manage opportunities," International Journal of Project Management, vol. 20, no. 3, pp. 235-240, 2002.

[26] Traker. http://physlets.org/tracker/. 


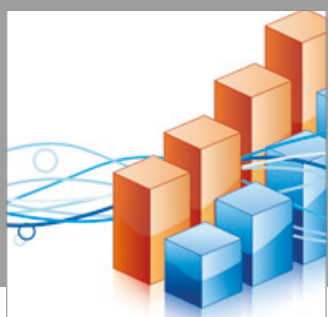

Advances in

Operations Research

vatersals

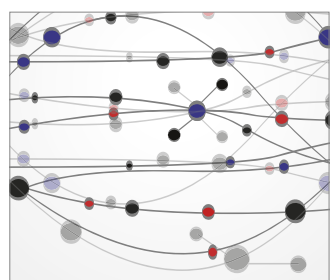

\section{The Scientific} World Journal
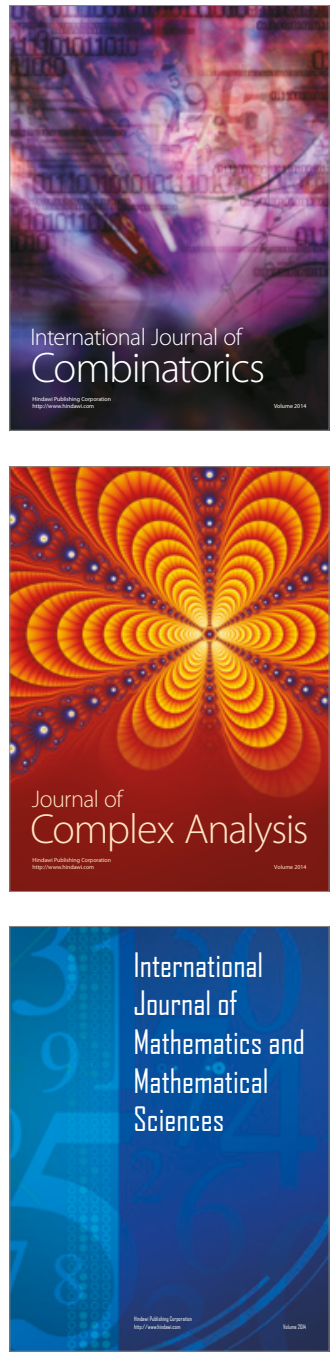
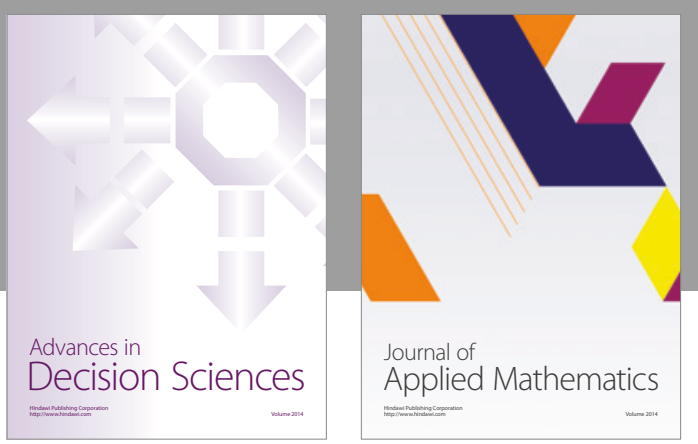

Algebra

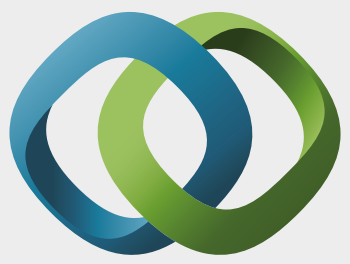

\section{Hindawi}

Submit your manuscripts at

https://www.hindawi.com
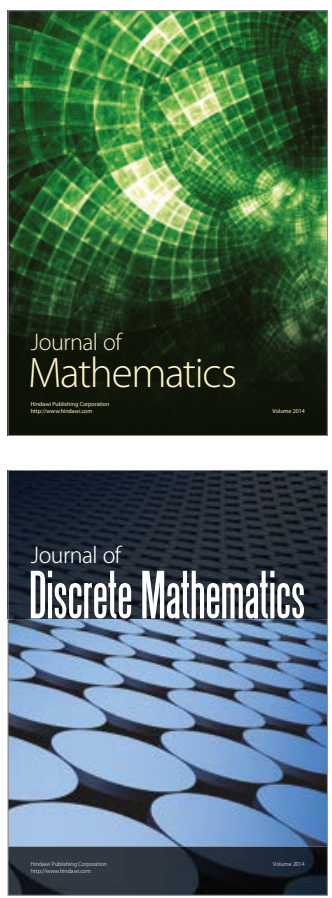

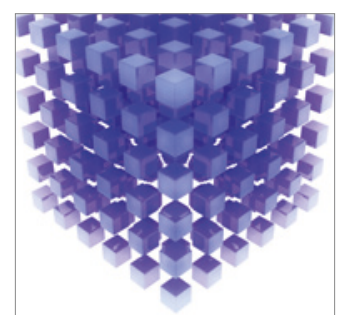

Mathematical Problems in Engineering
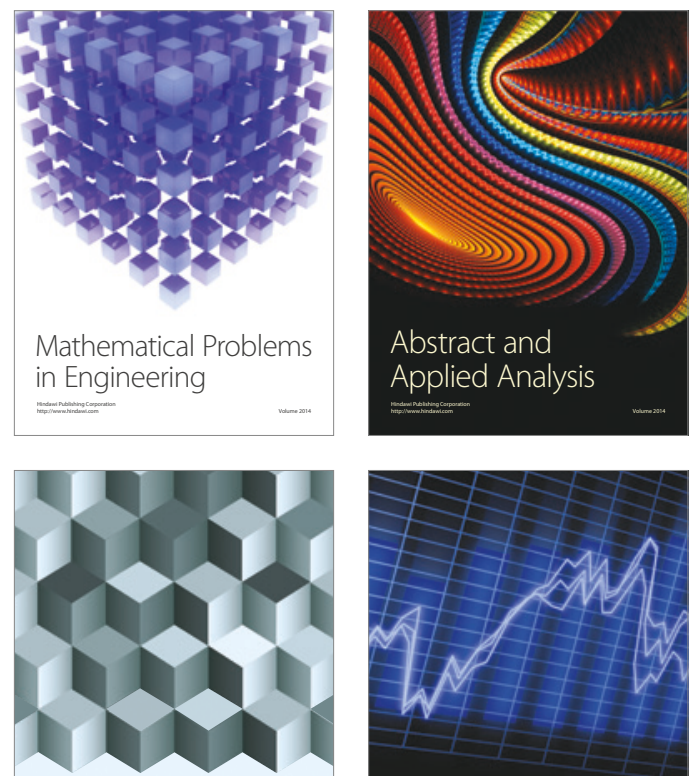

Journal of

Function Spaces

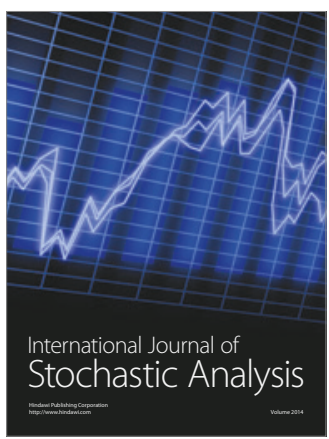

Probability and Statistics
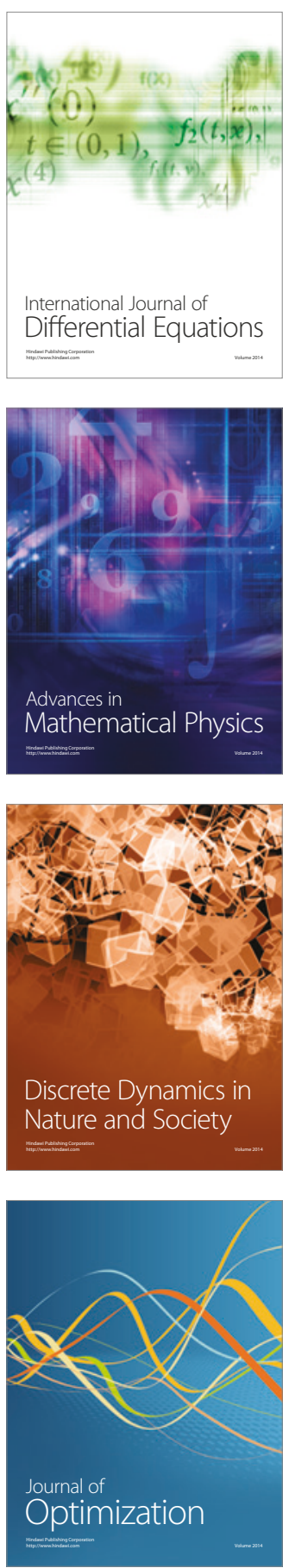